\title{
Autores y citas en contabilidad de gestión en revistas de lengua española
}

\author{
Lunkes, Rogério João* \\ Ripoll Feliu, Vicente M.** \\ da Rosa, Fabricia Silva***
}

\section{Resumen}

La formación de redes alienta y ayuda en el intercambio de tecnologías, experiencias e ideas entre los investigadores y las instituciones, a fin de generar mayor innovación y desarrollo del área. El objetivo es identificar y analizar las redes de los autores y las citas en contabilidad de gestión en las revistas electrónicas en lengua española. Esto permitirá comprobar el grado de centralización e integración, más allá del nivel de internacionalización de las publicaciones en contabilidad de gestión en las revistas de lengua española. Con este fin, se seleccionaron siete revistas de Lengua Española, disponible en la base de datos Latindex, y se analizaron 183 artículos de 2001 al 2010. Entre los principales resultados obtenidos, es pertinente señalar: (i) el limitado número de redes sociales en relación a los autores y una densa red en términos de citas, con el núcleo formado por Joan Amat Salas, Oriol Amat Salas y Ripoll Feliu. En el ámbito internacional una de las redes más importantes en cuanto a citas la lidera Robert Kaplan.

Palabras clave: Contabilidad de gestión, redes sociales, autores, citas, internacionalización.

\section{Citations and Authors in Management Accounting for Spanish Language Journals}

\begin{abstract}
Forming networks encourages and aids the exchange of technologies, experiences and ideas among researchers and institutions in order to generate greater innovation and development of the area. The objective of this study is to identify and analyze networks of authors and citations in man-

Recibido: 12-05-11. Aceptado: 27-03-12

* Post-Doctorado de la Universidad de Valencia. Profesor del Departamento de Ciencias Contables y Diploma de Postgrado en Contabilidad y Gestión, Universidad Federal de Santa Catarina de becas de la Capes - Proc. Nº1189/10-7. e-mail: lunkes@cse.ufsc.br.

** Doctor y profesor de la Universidad de Valencia. e-mail: Vicente.ripoll@uv.es.

*** Doctora de la Universidad Federal de Santa Catarina, patrocinados por el CNPq. e-mail: fabriciasrosa@hotmail.com.
\end{abstract}


Autores y citas en contabilidad de gestión en revistas de lengua española Lunkes, Rogério João; Ripoll Feliu, Vicente M. y da Rosa, Fabricia Silva

agement accounting for electronic journals in Spanish, making it possible to verify the degree of centralization and integration beyond the internationalization level of management accounting literature in Spanish-language magazines. To accomplish this, seven Spanish language magazines, available in the Latindex database, were selected; 183 articles from 2001 to 2010 were analyzed. The following principle results were noted: the limited number of social networks related to authors and a dense network in terms of citations, with a nucleus formed by Joan Amat Salas, Oriol Amat Salas and Ripoll Feliu. Internationally, one of the most important networks for citations is led by Robert Kaplan.

Keywords: Management accounting, social networks, authors, citations, internationalization

\section{Introducción}

En las últimas décadas, la contabilidad de gestión ha experimentado un cambio considerable, con la inclusión de nuevos temas de investigación (Johnson y Kaplan, 1987; Cooper, 1987; Kaplan, 1983, Hiromoto, 1988; Bromwich, 1990; Dent, 1990; Young y Selton, 1991; Kaplan y Norton, 1992; Shank y Govindarajan, 1993; Simons, 1995), revistas exclusivas en contabilidad de gestión, y especialmente los estudios con perspectivas multidisciplinar (Baiman, 1982; Covaleski et al., 1996; Hopwood y Shields, 1978; Macintoch y Scapens,1990, Kaplan y Norton, 2001). Esto se refleja principalmente en el florecimiento de costes basado en las actividades, gestión basada en las actividades, la contabilidad de gestión en Japón, gestión estratégica de costes, contabilidad de gestión estratégica, cuadro de mando integral y control estratégico.

Estas modificaciones y cambios no se perciben y se incorporan de manera lineal, si no que depende del nivel de desarrollo de la contabilidad de gestión en cada país. Una manera de entender el nivel de desarrollo de la contabilidad de gestión es el estudio de las redes sociales formadas por investigadores e instituciones en diferentes partes del mundo. Se entiende que no hay manera de producir gran innovación y la difusión de nuevos conocimientos en contabilidad de gestión sin el desarrollo de redes sociales confiables, que integraría a los investigadores e instituciones de diferentes países, con pensamientos, culturas y niveles de desarrollo diferentes. La colaboración entre los investigadores y las instituciones se percibe como algo positivo y debe fomentarse (Katz y Marting, 1997), porque compartir tecnologías, experiencias e ideas entre los investigadores y las instituciones, genera un aumento significativo y mejoras en todos los involucrados (Da Cruz et al., 2010). Estas redes pueden estar formadas por investigadores e instituciones de diferentes países, con un marcado grado de internacionalización.

Las redes sociales son estructuras sociales integradas por personas e instituciones vinculadas a uno o más tipos de relaciones que comparten valores y objetivos comunes. Una de las definiciones fundamentales de las redes es su apertura y la porosidad, lo que permite las relaciones no-jerárquicas y horizontales entre los participantes. Uno de los puntos comunes entre los distintos tipos de redes sociales es el intercambio de información, conocimientos, intereses y esfuerzos en pos de objetivos comunes (Duarte y Flei, 2008; Freeman, 2006). 
Varios estudios han sido desarrollados con el fin de evaluar la producción científica de contabilidad de gestión (Carvalho et al., 2010; Nascimento, Junqueira y Martins, 2010; Cardoso, Pereira y Guerreiro, 2007; Mendonca, Riccio y Sakata, 2009; Beuren, Schlindwein y Pasqual, 2007; Maloni, Carter y Carr, 2009; Hesford y Potter, 2010; Alcouffe, Berland y Levant, 2008; Groot y García-Valderrama, 2006; Valacich, et al 2006; Tahai y Meyer, 1999; Engwall, 1998; White, 1996, Kirkpatrick y Locke, 1992). Sin embargo, en el área de Contabilidad de Gestión ha pocos estudios sistemáticos sobre redes de autores y citas (Casanueva, Escobar y Larrinaga, 2007; Hesford et al., 2007; Alcouffe, Berlande e Levant, 2008; Lunkes, Ripoll y Rosa, 2011).

El análisis de redes sociales centra la atención en los autores e instituciones que interactúan entre sí (Galaskiewicz y Wasserman, 1994). Por lo tanto, la identificación y análisis de redes de investigación en contabilidad de gestión, puede proporcionar información importante sobre sus niveles de integración y desarroIlo. El objetivo del presente trabajo es identificar y analizar las redes de los autores y las citas en contabilidad de gestión en las revistas electrónicas en lengua española.

\section{Redes sociales}

Red social se puede definir como un conjunto de relaciones o ligaciones sociales entre un conjunto de atores (Emirbayes y Goodwin, 1994).

En cuanto a la estructura de las relaciones, Rossoni y Machado-da-Silva (2007) afirman que las relaciones socia- les juegan un papel fundamental en la construcción y significado de una investigación en cualquier campo de la ciencia. Scott (2008) sostiene que las redes sociales en que las instituciones e investigadores están inmersas, actúan como conductores del conocimiento. Owen-Smith y Powell (2008) argumentan que cualquier esfuerzo dirigido a entender los procesos institucionales tiende a ser más fructífero con la consideración de la perspectiva de redes.

La estructura de las relaciones entre los investigadores de un determinado campo, pueden condicionar las decisiones científicas, incrementar el grado de colaboración que se produce entre científicos, e incluso establecer una red de contactos que permite conectar a investigadores de cualquier parte del mundo en pocos pasos (Newman, 2001; Barabasi et al., 2002; Casanueva, Escobar y Larrinaga, 2007).

Según Casanueva, Escobar y Larrinaga (2007) la perspectiva de red supone una visión diferente dentro del campo de las ciencias sociales debido a su presuposición acerca de la importancia de las relaciones entre las unidades que interactúan, además de sus propias características.

El análisis de redes permite una mayor comprensión de los autores que colaboran, de los grupos sociales que emergen de las relaciones de colaboración, el papel desempeñado por los autores, y de los efectos de su pertenencia a determinadas instituciones y revistas (Acedo et al., 2006). En este sentido, Kuhn (1962) considera que los determinantes de la conducta científica que explican el cambio científico son factores so- 
Autores y citas en contabilidad de gestión en revistas de lengua española Lunkes, Rogério João; Ripoll Feliu, Vicente M. y da Rosa, Fabricia Silva

ciológicos como la autoridad, el poder y/o los grupos de referencia, más que factores cognitivos.

Para Barabasi et al. (2002), las investigaciones de red de co-autoría de los científicos representa un prototipo de complejas redes en evolución. De los estudios al respecto de la evolución de la red social de colaboración científico se infiere la dinámica y los mecanismos estructurales que rigen del evolución y la topología de este complejo sistema, donde hay la determinación de la conducta observada y la ampliación topología de la red.

En el área de gestión, la investigación hecha por Acedo et al. (2006) en revistas científicas muestra un progresivo crecimiento en el número de co-autores de documentos y que los autores con más publicaciones y con una mayor influencia son prácticamente interconectados a través de la red de co-autoría, con un gran número de directos enlaces.

Así, los diferentes estudios a respecto de las redes (Brown y Gardner,1985a y 1985b; Brown et al., 1987; Salas et al., 1998; Newman, 2001; Acedo et al., 2006; Moody, 2004, Freeman, 2000; Molina, Muñoz y Domenech, 2002; Larrinaga, 2005; Escobar, Lobo y Martínez, 2005; Casanueva, Escobar y Larrinaga, 2007; Hesford et al., 2007; Alcouffe, Berlande y Levant, 2008), ayudan a comprender el perfil de los investigadores, las interconexiones hechas para desarrollar las investigaciones y los avances científicos conquistados por las distintas redes al largo de los años. Permitiendo identificar las tendencias, las oportunidades y los puntos fuertes que están investigados.
Pero hay que tener en cuenta que los estudios sobre redes en contabilidad son recientes (Molina, Muñoz y Domenech, 2002; Acedo et al, 2004) y por lo tanto, es al mismo tiempo un campo fértil porque hay mucho que investigar, así como también lo relativo al proceso de selección de criterios y métodos de análisis de las redes no están totalmente consolidados.

\section{Revistas de lengua españolas y la contabilidad de gestión}

La selección de las revistas en contabilidad de gestión se basa en su inclusión en Latindex, este es un sistema de información sobre las revistas de investigación científica, técnico-profesionales y de divulgación científica y cultural que se editan en los países de América Latina, el Caribe, España y Portugal. Se consideró un período de 10 años (2001 a 2010).

Se utilizó la base de datos Latindex (búsqueda de catálogo) para hacer la selección de las revistas y se seleccionaron aquellas correspondientes con el área de conocimiento de "Ciencias Sociales", el cual contenía 2.874 revistas. Desde esta área, se seleccionó el ámbito de la contabilidad, el cual está formado por 31 revistas. De esta selección inicial se excluyeron las revistas publicadas en lengua portuguesa (15 revistas) y las que no ofrecen los elementos para su consulta por medios electrónicos (9 revistas). Para la selección de las revistas españolas también se consideró la inclusión IN-RECS Índice (impacto de las revistas españolas de ciencias sociales) elaborado por el grupo de investigación EC3 de Evaluación de la 
Ciencia y de la Comunicación de Universidad de Granada.

Por consiguiente se seleccionaron siete revistas que ofrecen artículos en su totalidad en los medios de comunicación electrónicos. Estas son: Revista de Contabilidad (España), Revista Iberoamericana de Contabilidad de Gestión (España), Contabilidad y Negocios (Perú), Economía, Gestión y Desarrollo (Colombia), Semestre Económico (Colombia), Actualidad Contable Faces (Venezuela), Contaduría y Administración (México).

Siendo la Revista Iberoamericana de Contabilidad de Gestión la única publicación donde todos los artículos son de contabilidad de gestión, lo que hizo que todas las publicaciones se incluyeran en la búsqueda.

En la Tabla 1, aparecen los 183 artículos seleccionados relacionados con la contabilidad de gestión. Esta selección se realizó de forma manual mediante la lectura del resumen y, cuando procedía, se llevaba a cabo una revisión del artículo en su totalidad. No se han tenido en cuenta los artículos relacionados con notas de investigación, reseñas de libros y tesis, editoriales, entre otros. Los resultados muestran que aproximadamente el $23 \%$ de los artículos publicados durante el periodo están relacionados con la contabilidad de gestión. Sin embargo, si se extrae de la muestra la revista RICG (exclusiva de contabilidad de gestión), este porcentaje se reduce aproximadamente el $10 \%$, lo que indica un bajo nivel de publicación. Es importante destacar que, a pesar de que algunas revistas tienen un número reducido de artículos, hay un aumento en términos absolutos de las publicaciones en el periodo considerado, impulsado principalmente por la aparición de la revista especializada en contabilidad de gestión.

Para mejorar el análisis de redes de autores y de citas en contabilidad de gestión, se identifican algunas características de las revistas, artículos y autores.

Tabla 1

Resultados de las revistas seleccionadas

\begin{tabular}{lcc}
\hline \multicolumn{1}{c}{ Revistas Seleccionadas } & $\begin{array}{c}\text { Total de artículos } \\
(2001 \text { el 2010) }\end{array}$ & $\begin{array}{c}\text { Artículos Seleccionados } \\
(2001 \text { el 2010) }\end{array}$ \\
\hline RC-Revista de Contabilidad (España) & 113 & $15(13 \%)$ \\
RICG-Revista Iberoamericana de Contabilidad de & $116^{*}$ & $116(100 \%)$ \\
Gestión (España) & & $8(15 \%)$ \\
RCN-Contabilidad y Negocios (Perú) & $52^{* *}$ & $4(5 \%)$ \\
REGD-Economía, Gestión y Desarrollo (Colombia) & $86^{*}$ & $3(3 \%)$ \\
RSE-Semestre Económico (Colombia) & $86^{* * *}$ & $27(20 \%)$ \\
RACF-Actualidad Contable Faces (Venezuela) & $134^{*}$ & $10(5 \%)$ \\
RCA-Contaduría y Administración (México) & 193 & $183(23 \%)$ \\
Total & 780 &
\end{tabular}

* Disponible a partir de 2003. ** Disponible a partir de 2006. ${ }^{* * *}$ Disponible a partir de 2004

Fuente: Elaboración propia. 
Autores y citas en contabilidad de gestión en revistas de lengua española Lunkes, Rogério João; Ripoll Feliu, Vicente M. y da Rosa, Fabricia Silva

El análisis de las características de las revistas es una manera de entender las principales tendencias y líneas editoriales. Para ello los artículos fueron clasificados en los ítems siguientes: costes, planificación y control y otras cuestiones relacionadas con la contabilidad de gestión. Los resultados muestran que el $38 \%$ de los artículos se centran en otras cues- tiones de contabilidad de gestión. Planificación y control el $32 \%$ y el área de los costes, el $30 \%$, tal y como aparece en la Tabla 2.

Los artículos se clasifican también según el método de investigación empleado. De acuerdo a la Tabla 3, la clasificación de los artículos muestra un predominio de los métodos de estudio de caso

Tabla 2

Clasificación de los artículos de acuerdo con las revistas y temas

\begin{tabular}{lcccr}
\hline Revistas & Costes & $\begin{array}{c}\text { Planificación y } \\
\text { Control }\end{array}$ & Otros & \multicolumn{1}{c}{ Total } \\
\hline RC & $3(20 \%)$ & $5(50 \%)$ & $7(70 \%)$ & $15(100 \%)$ \\
RICG & $34(29 \%)$ & $40(35 \%)$ & $42(36 \%)$ & $116(100 \%)$ \\
RCN & $1(12,5 \%)$ & & $7(87,5 \%)$ & $8(100 \%)$ \\
REGD & $1(25 \%)$ & $1(25 \%)$ & $2(50 \%)$ & $4(100 \%)$ \\
RSE & $2(67 \%)$ & & $1(33 \%)$ & $3(100 \%)$ \\
RACF & $11(41 \%)$ & $11(41 \%)$ & $5(18 \%)$ & $27(100 \%)$ \\
RCA & $3(30 \%)$ & $2(20 \%)$ & $5(50 \%)$ & $10(100 \%)$ \\
Total & $55(30 \%)$ & $59(32 \%)$ & $69(38 \%)$ & $183(100 \%)$ \\
\hline
\end{tabular}

Fuente: Elaboración propia.

Tabla 3

Clasificación de los artículos de acuerdo con las revistas y métodos

\begin{tabular}{|c|c|c|c|c|c|c|c|}
\hline Revistas & Archivo & $\begin{array}{l}\text { Estudio de } \\
\text { Caso }\end{array}$ & $\begin{array}{l}\text { Experimen- } \\
\text { tales }\end{array}$ & $\begin{array}{l}\text { Estudio de } \\
\text { Campo }\end{array}$ & Revisión & Survey & Total \\
\hline $\mathrm{RC}$ & & $6(40 \%)$ & & $3(20 \%)$ & $1(7 \%)$ & $5(33 \%)$ & $15(100 \%)$ \\
\hline RICG & $1(1 \%)$ & $41(35 \%)$ & $2(2 \%)$ & $26(22 \%)$ & $32(28 \%)$ & $14(12 \%)$ & $116(100 \%)$ \\
\hline $\mathrm{RCN}$ & & $4(50 \%)$ & & $1(12,5 \%)$ & $3(37,5 \%)$ & & $8(100 \%)$ \\
\hline REGD & & $2(50 \%)$ & & & $2(50 \%)$ & & $4(100 \%)$ \\
\hline RSE & & & & $2(77 \%)$ & $1(33 \%)$ & & $3(100 \%)$ \\
\hline RACF & & $5(18,5 \%)$ & & $5(18,5 \%)$ & $14(52 \%)$ & $3(11 \%)$ & $27(100 \%)$ \\
\hline $\mathrm{RCA}$ & & $1(10 \%)$ & & $2(20 \%)$ & $4(40 \%)$ & $3(30 \%)$ & $10(100 \%)$ \\
\hline Total & $1(0,50 \%)$ & $59(32 \%)$ & $2(1 \%)$ & $39(21,5 \%)$ & $57(31 \%)$ & $25(14 \%)$ & $183(100 \%)$ \\
\hline
\end{tabular}

Fuente: Elaboración propia. 
con $32 \%$, revisión con $31 \%$ y estudios de campo con $21,5 \%$.

Los resultados muestran una amplia variedad de métodos empleados y no se ha diagnosticado la concentración de un método de investigación único en las revistas analizadas.

La investigación sobre costes es en su mayor parte realizada a través del estudio de caso (33\%). En los trabajos de planificación y control se utiliza el método de revisión, y en otros temas, también se emplea este mismo método con $35 \%$, tal y como aparece en la Tabla 4.
En cuanto al número de autores por artículo, el $48 \%$ tiene sólo un autor por trabajo. En el $32 \%$ de los casos aparecen dos autores. En el $15 \%$ tres y un $5 \%$ sólo para cuatro autores. Estos datos se presentan en la Tabla 5. Ninguna publicación cuenta con cinco o más autores por artículo.

\section{Redes de autores y de citas en contabilidad de gestión}

A continuación se identifican las redes sociales a la que forman parte los autores y citas.

\section{Tabla 4}

Clasificación de los artículos según tema y método

\begin{tabular}{lcccc}
\hline \multicolumn{1}{c}{$\begin{array}{c}\text { Métodos de } \\
\text { Investigación }\end{array}$} & Costes & $\begin{array}{c}\text { Planificación y } \\
\text { Control }\end{array}$ & Otros & Total \\
\hline Archivo/documento & & $1(2 \%)$ & $1(1 \%)$ \\
Estudio de Caso & $33(60 \%)$ & $15(25 \%)$ & $11(16 \%)$ & $59(32 \%)$ \\
Experimentales & $1(2 \%)$ & & $1(1 \%)$ & $2(1 \%)$ \\
Estudio de Campo & $5(9 \%)$ & $13(22 \%)$ & $21(30 \%)$ & $39(21 \%)$ \\
Revisión & $12(22 \%)$ & $21(36 \%)$ & $24(35 \%)$ & $57(31 \%)$ \\
Survey & $4(7 \%)$ & $9(15 \%)$ & $12(17 \%)$ & $25(14 \%)$ \\
Total & $55(100 \%)$ & $59(100 \%)$ & $69(100 \%)$ & $183(100 \%)$ \\
\hline
\end{tabular}

Fuente: Elaboración propia.

Tabla 5

Número de autores por artículo

\begin{tabular}{lcccccccc}
\hline Autores & RC & RICG & RCN & REGD & SER & RACF & RCA & Total \\
\hline 1 & $3(20 \%)$ & $50(43 \%)$ & $5(62 \%)$ & $2(50 \%)$ & $1(33 \%)$ & $20(74 \%)$ & $7(70 \%)$ & $88(48 \%)$ \\
2 & $6(40 \%)$ & $45(39 \%)$ & $1(13 \%)$ & & $2(67 \%)$ & $3(11 \%)$ & $2(20 \%)$ & $59(32 \%)$ \\
3 & $5(33 \%)$ & $16(14 \%)$ & $2(25 \%)$ & $1(25 \%)$ & & $3(11 \%)$ & $1(10 \%)$ & $28(15 \%)$ \\
4 & $1(7 \%)$ & $5(4 \%)$ & & $1(25 \%)$ & & $1(4 \%)$ & & $8(5 \%)$ \\
5 o más & & & & & & & & \\
Total & $15(100 \%)$ & $116(100 \%)$ & $8(100 \%)$ & $4(100 \%)$ & $3(100 \%)$ & $27(100 \%)$ & $10(100 \%)$ & $183(100 \%)$ \\
\hline
\end{tabular}

Fuente: Elaboración propia. 
Autores y citas en contabilidad de gestión en revistas de lengua española Lunkes, Rogério João; Ripoll Feliu, Vicente M. y da Rosa, Fabricia Silva

\subsection{Redes de los autores}

Una forma de analizar las redes en contabilidad de gestión es identificar las asociaciones entre los autores en las publicaciones. Esto le permite crear matrices o mapas de relaciones, la identificación del autor y la red central de los autores participantes (Molina, Muñoz y Domenech, 2002; Acedo et al., 2004).

Como muestra el Diagrama 1, se observan las interacciones de la red principal, constituida por dieciocho relaciones, cuyo eje central es el profesor Ripoll
Feliu de la Universidad de Valencia. Esta red cuenta con la participación de profesores de diferentes países como Brasil, Cuba, Venezuela y México. Esta red central tiene un enlace con la segunda formada en el centro por el profesor Oriol Amat Salas. Estos dos grupos tienen conexiones privilegiadas, también llamados de colegios invisibles (Crane, 1972). También hay otros pequeños grupos aislados como el formado por el Prof. Domingo García Pérez de Lema de la Universidad Politécnica de Cartagena (España).

\section{Diagrama 1 \\ Principales autores de las redes sociales en revistas de la lengua española}

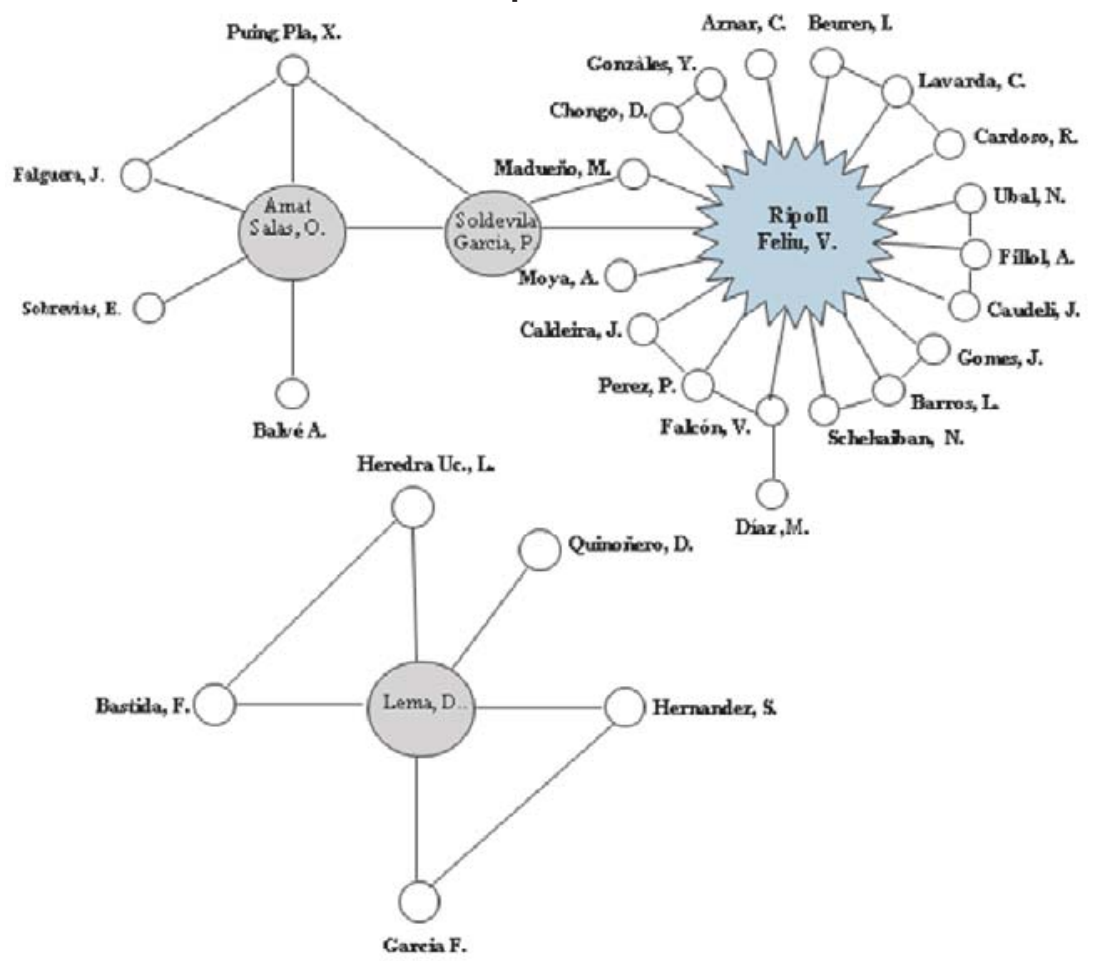

Fuente: Elaboración propia. 
Cabe señalar dos redes más pequeñas aisladas. La primera es compuesta por la profesora María Luisa Vélez Elorza de la Universidad de Cádiz y el profesor José Manuel Sánchez Vázquez de la Universidad Pablo de Olavide, y la segunda red, es formada por Ana Isabel Zardoya-Alegría de la Universidad de Zaragoza.

\subsection{Redes sociales por citas}

En el estudio de las redes sociales es importante examinar las referencias o citas con el fin de identificar las relaciones entre los autores, los temas y las instituciones (Salas et al., 1998; Escobar, Lobo y Martínez, 2005; Larrinaga, 2005; Moody, 2004). Se analizaron 5.106 citas relacionadas con los 183 artículos de la muestra. Para ello, se utilizó un programa informático que permite identificar y analizar las redes sociales. Tal y como aparece en la Tabla 6, la Revista de Contabilidad es la revista con mayor número promedio de citas por artículo (45), mientras que la RCN es la que tiene menos referencias (6) por artículo.

De acuerdo con la Tabla 7, Robert Kaplan es el autor más referenciado con 166 citas, 74 de ellos junto a David Norton y 23 con Robert Cooper. En los últimos años se ha producido un cambio en el contexto de la contabilidad de gestión, el cual ha dado lugar a que muchas de las referencias o citas sobre las nuevas tendencias en este contexto y por el lado de las publicaciones en lengua española, se focalicen en Kaplan y Norton, por un lado, y Kaplan y Cooper, por el otro.
Tabla 6 Número de citas por artículo

\begin{tabular}{lrcc}
\hline Revistas & $\begin{array}{c}\mathrm{N}^{\circ} \text { de } \\
\text { Autores }\end{array}$ & $\begin{array}{c}\text { Media de las } \\
\text { citas p/artículo }\end{array}$ & Ranking \\
\hline RC & 680 & 45 & 1 \\
RICG & 3.586 & 31 & 2 \\
RCN & 45 & 6 & 7 \\
REGD & 89 & 22 & 3 \\
RSE & 45 & 15 & 6 \\
RACF & 472 & 17 & 5 \\
RCA & 189 & 19 & 4 \\
Total & 5.106 & 28 & - \\
\hline
\end{tabular}

Fuente: Elaboración propia.

AECA (Asociación Española de Contabilidad y Administración de Empresas) es la segunda referencia más utilizada, con 75 citas. Entre los autores españoles destacan Lizcano con 51, Ripoll Feliu con 48 y Castelló Taliani con 27 citas, tal y como aparece en la Tabla 7 .

La utilización de las obras publicadas por AECA es una particularidad de las publicaciones en español. Esto demuestra la calidad y la influencia de estas obras y el notable trabajo realizada por la Asociación en su publicación, que merece alabanza, y es un ejemplo para otros países que tienen una carencia de la literatura de contabilidad y gestión.

De los autores extranjeros que aparecen en la Tabla 7 , se pone de manifiesto que autores clásicos como Scapens centran su investigación sobre temas centrales como las teorías de la contabilidad de gestión, conceptos y métodos de investigación; Gray tiene su línea de investigación centrada en la contabilidad internacional y sus efectos en las distintas cultu- 
Autores y citas en contabilidad de gestión en revistas de lengua española Lunkes, Rogério João; Ripoll Feliu, Vicente M. y da Rosa, Fabricia Silva

Tabla 7

Autores con más de diez citas

\begin{tabular}{lclc}
\hline \multicolumn{1}{c}{ Autores } & $\mathrm{N}^{\circ}$ de Citas & \multicolumn{1}{c}{ Autores } & No de Citas $^{\circ}$ \\
\hline Kaplan, R. & 166 & Johnson, H. & 25 \\
AECA & 75 & Amat Salas, O. & 24 \\
Norton, D. & 74 & Innes, J. & 24 \\
Cooper, R. & 64 & Chenhall, R. & 20 \\
Lizcano, J. & 51 & Amat Salas, J. & 20 \\
Ripoll, V. & 48 & Fernández, A. & 19 \\
Scapens, R. & 32 & Hopwood, A. & 18 \\
Gray, S. & 27 & Drucker, P. & 16 \\
Castelló Taliani, E. & 27 & Shields, M. & 14 \\
Ittner, C. & 26 & Yin, R. & 13 \\
Horngren, C. & 26 & Porter, M. & 11 \\
\hline Fuente: Elaboración propia. & & &
\end{tabular}

ras; Ittner es un autor que trabaja con los problemas de coste y el $A B C$, así como la evaluación del desempeño; Horngren tiene su foco principal en la contabilidad de costes; Johnson tiene una obra en colaboración con Kaplan, que revolucionó la contabilidad de gestión.

Innes lleva a cabo estudios sobre el sistema de costes y gestión basado en las actividades, Chenhall sobre trabajos de contabilidad de gestión en el contexto de la organización; Hopwood tiene estudios relacionados con el papel de la contabilidad en las organizaciones; Shields dirige su investigación a los sistemas de control; Yin estudia los métodos de investigación. Se debe destacar que autores como el padre de la administración, Peter Drucker y Michael Porter, con su línea de investigación centrada en la ventaja competitiva, que en la literatura anglosajona de contabilidad de gestión son muy poco utilizados al considerarlos generalistas y más cerca del campo de la administración, en las referencias o citas de los trabajos en lengua española son utilizados por muchos de los autores.

Si se comparan los resultados de este trabajo con los que se ponen de manifiesto en Hesford et al. (2007) sobre revistas de lengua inglesa en el período comprendido entre 1981 y 2000 , se encuentran similitudes en cuanto a los autores más citados, ya que seis de los veinte (Kaplan, Shields, Hopwood, Chenhall, Cooper y Scapens) tienen referencias tanto en publicaciones en inglés como en español. Los autores Young, Merchant, Govindarajan e Banker en la investigación de Hesford et al. (2007) se encuentran entre los más citados, y también son citados en la literatura en español. La excepción recae en Baiman y Brownell que tiene un gran número de citas en lengua inglesa y en las revistas en lengua española prácticamente no se hace referencia. 
En el Diagrama 2, las redes sociales formadas por las citas de revistas en español forman una red que tiene como núcleos a Ripoll Feliu, Joan Amat Salas y Oriol Amat Salas. Esta red social está relacionada de diferentes maneras con Emma Castelló Taliani, Jesús Lizcano Álvarez, Felipe Blanco Ibarra, Tomas Balada Ortega y José Álvarez López.

Esta red social se compone de tres núcleos y cuenta con la participación de diferentes autores e instituciones, lo que demuestra la fuerza de la literatura española. La red que se forma en el centro por el profesor Oriol Amat Salas tiene una gran interacción entre los participantes y autores de diferentes países.

Estas redes son fundamentales para el avance de la contabilidad de ges- tión en los países subdesarrollados o en desarrollo, ya que permite el intercambio de conocimientos y la formación y cualificación de los docentes en el área. Los autores españoles tiene un papel clave en la difusión de nuevos conocimientos, tecnologías y experiencias en países de América Latina y la creación de redes con los autores locales es una manera de desarrollar esta integración. Estas asociaciones trabajan como conductores en la difusión de las innovaciones y los cambios efectuados en contabilidad de gestión.

Con respecto a autores extranjeros existe la formación de una red de citas en el centro de Robert Kaplan, de acuerdo con el Diagrama 3. Esta red está formada básicamente por autores estadounidenses, lo que demuestra la

\section{Diagrama 2}

Redes sociales formadas por las citas en revistas

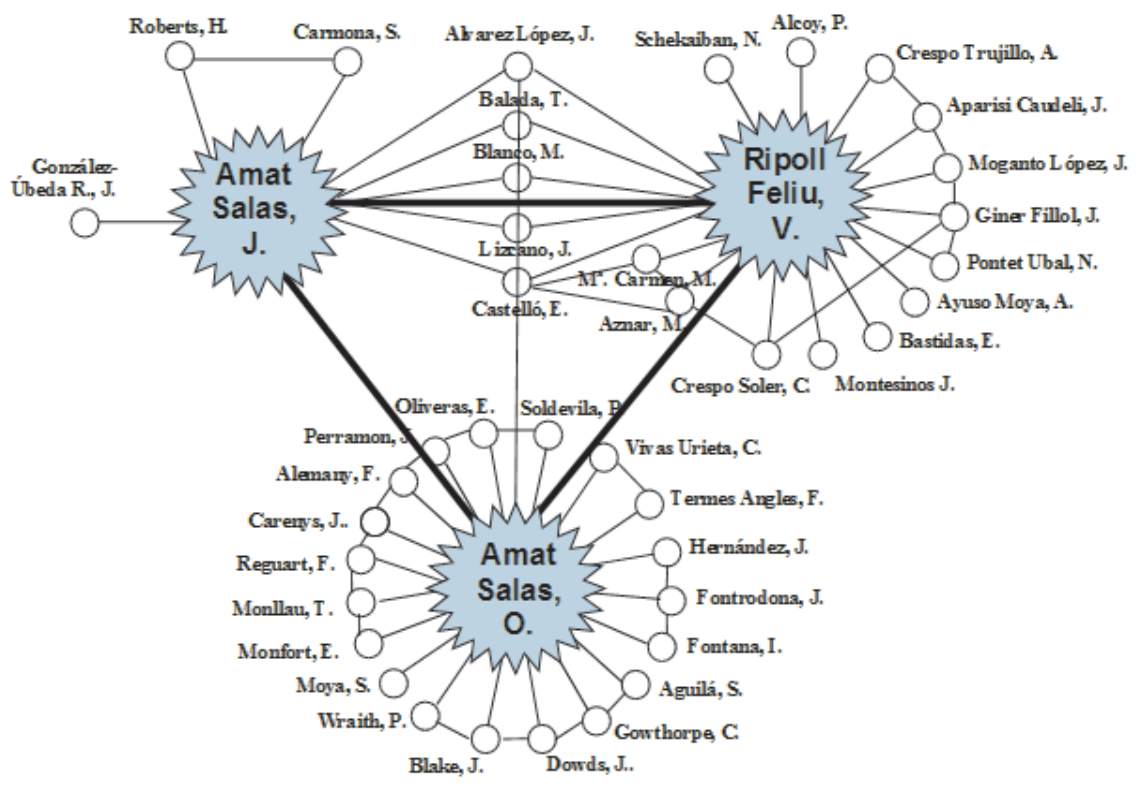

Fuente: Elaboración propia. 
Autores y citas en contabilidad de gestión en revistas de lengua española

Lunkes, Rogério João; Ripoll Feliu, Vicente M. y da Rosa, Fabricia Silva

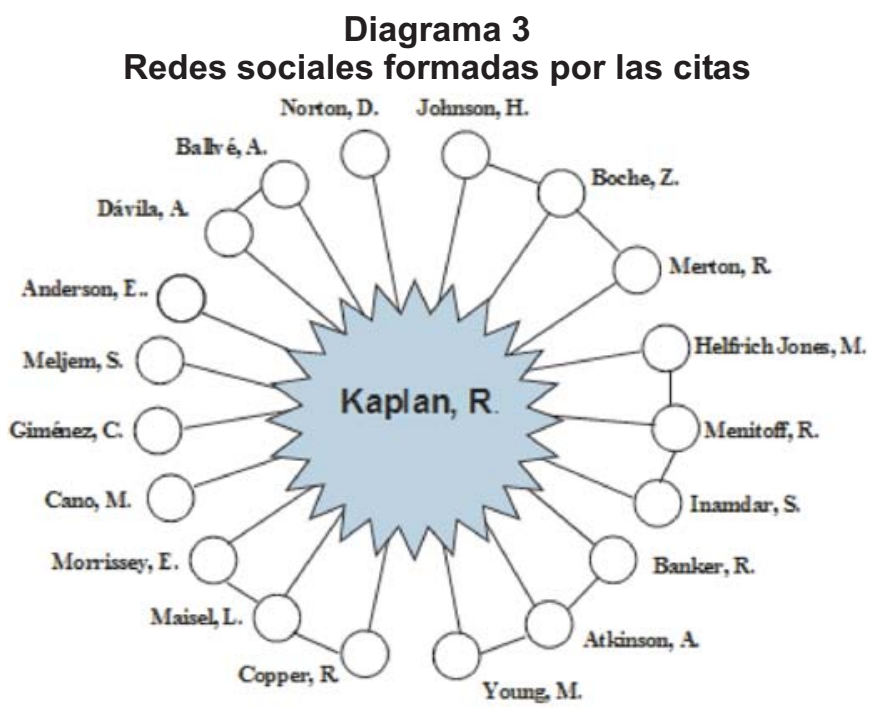

Fuente: Elaboración propia.

influencia de este país en la literatura de la lengua española.

La red formada principalmente por autores norteamericanos no se comunican con la red de autores en español, formado principalmente por autores españoles. Esto demuestra que las asociaciones entre autores españoles y los americanos son muy escasas y que podría ampliarse, con el objetivo de intercambiar experiencias y conocimientos. Según Atkinson et al. (1997), los autores europeos dependen en gran medida de los aspectos sociológicos de la contabilidad de gestión y su papel en las organizaciones. Las publicaciones de EE.UU. se basan en temas económicos y estudios con aplicaciones de métodos experimentales y un fuerte predominio de métodos estadísticos.

Para Hesford et al. (2007), Scapens (2004) y Shields (1997), esto se debe a la formación de los programas norteamericanos de doctorado en contabilidad, ya que tienen en su currículo muchas disciplinas estrechamente relacionadas con la economía y centrada en métodos cuantitativos. Esta interacción debería favorecer el desarrollo de las diferentes escuelas o corrientes doctrinales en contabilidad de gestión.

En la investigación de Hesford et al. (2007), en el período 1981 el 2000 Kaplan ya era el autor más citado, pero no tiene, en ese periodo, una red social extensa. En cambio, en este trabajo, Kaplan, tal y como se pone de manifiesto en el Diagrama 3, sí que mantiene una red social extensa o amplia, probablemente debido al éxito de la metodología del Balanced Scorecard, lo cual hizo que aumentara significativamente su red social, debido a la aceptación de la herramienta. Para Wasserman y Faust (1994), Kaplan es un "autor de prestigio". 


\section{Conclusiones}

Este artículo ha alcanzado su objetivo con la identificación y el análisis de redes de autores y citas en revistas electrónicas de lengua española, con la conclusión de que los profesores Oriol Amat Salas de la Universidad Pompeu Fabra y Ripoll de la Universidad de Valencia son los autores que tienen la mayor densidad en la red de los autores. La encuesta también diagnosticó la formación de pequeñas redes locales y no se ha detectado ninguna red formada por autores de América Latina de habla española, excepto la participación de estos autores en las redes formadas por autores españoles.

El análisis de las citas puso de manifiesto la formación de una densa red social con tres núcleos, teniendo en el centro a Oriol Amat Salas, a Ripoll Feliu y a Amat Salas. Esta red es más amplia que la formada por autores Americanos. En el trabajo de Hesford et al. (2007) en revistas de lengua inglesa, la mayor red estaba formada por 25 autores, esto puede deberse a una mayor interacción y a la amplitud de los autores españoles, es decir, los americanos escriben poco con investigadores de otros países, en cambio en España, no ocurre lo mismo. Los investigadores, realizan trabajos con gente de otros países, ya sean americanos, brasileños, alemanes o franceses. Esto podríamos interpretarlo de dos formas distintas. En la primera entendemos que se crea polaridad, es decir, no limita las posibilidades. En la segunda, que se profundiza en un tema específico de investigación, lo que limita la creación de centros de referencia.
Los americanos consideran que publicar con muchos autores no conduce a crear, por parte del investigador, un tema de investigación central. Es por ello que consideran a la contabilidad de gestión como un área de investigación, no como un tema como suele ocurrir en las revistas de lengua española. El investigar un área y no un tema puede llevar a que no se profundice mucho en la investigación.

Las citas y la formación de una red con Robert Kaplan en el centro, muestra una forma de influencia de la literatura americana sobre las publicaciones en lengua española. Esto comprueba que los determinantes de la conducta científica que explican la formación de las redes son los factores sociológicos (Kuhn, 1962).

La escasa integración de las redes en los EE.UU. puede estar relacionada con la manera española de hacer la investigación. Mientras que los primeros se basan en gran medida en temas económicos y estudios con aplicación de métodos experimentales y un predominio de métodos estadísticos, los estudios de los españoles de la contabilidad de gestión tienden a utilizar disciplinas sociológicas, aplican estudios de casos y de campo y utilizan análisis cualitativo.

No se diagnosticó una red social formada por temas de investigación específicos. Esta formación de redes es crucial ya que permite a los investigadores centrarse en temas determinados de investigación y crear centros de referencia en un campo de la contabilidad de gestión. El proceso de internacionalización se ha demostrado que es fuerte en relación a las citas y en cambio es débil 
Autores y citas en contabilidad de gestión en revistas de lengua española Lunkes, Rogério João; Ripoll Feliu, Vicente M. y da Rosa, Fabricia Silva

en relación a los autores, es decir la internacionalización se da por las citas realizadas pero no por las relaciones entre los autores de diferentes países. Por ejemplo, un determinado autor español, cita a autores americanos, pero en raras ocasiones este autor escribe con un autor americano.

Estas conclusiones se ven limitadas por las características propias de la investigación, por el contexto en que se ha desarrollado y por la escasa representación de las revistas españolas de contabilidad en el Journal Citation Reports (JCR) u otro índice que calcule el factor de impacto. Cómo se optó utilizar una base de datos que abarcara el mayor número de revistas en lengua española, al mismo tiempo que buscábamos tener acceso al contenido de las mismas, dado que los artículos no están totalmente disponibles en medios electrónicos y las revistas de contabilidad no están indexadas en bases de datos, donde pueden ser consultados.

\section{Referencias bibliográficas}

Acedo, Francisco José; Barro, Carmem; Casanueva Rocha, Carmen; Galpan, José Luis (2004). Co-Authorship in Management and Organizational Studies: An Empirical and Network Analysis. Journal of Management Studies, Año 43, № 5, United Kingdom, Durham Business School, Durham University, pp. 957-983.

Alcouffe, Simon; Berlandee, Nicolas; Levant, Yves (2008). Actor-networks and the diffusion of management accounting innovations: A comparative study. Management Accounting $\mathbf{R e}$ search, Año 19, № 1, United States of
America, American Accounting Association, pp: 1-17.

Atkinson, Anthony; Balakrishnan, Ramji; Booth, Peter; Cote, Jane M.; Groot, Tom; Malmi, Teemu; Roberts, Hanno; Uliana, Enrico; Wu, Anne (1997). New Directions in Management Accounting Research. Journal of Management Accounting Research, $\mathrm{N}^{\circ} 9$, United Kingdom. pp 79-108.

Baiman, Stanley (1982). Agency research in managerial accounting: a survey. Journal of Accounting Literature, № 1, United States of America, Warrington College of Business Administration, University of Florida, pp 154-213.

Barabási, Arbert László; Jeong, Hawoong; Ravasz, Erzsébet; Néda, Zoltán; Vissek, Tomás; Schubert, András (2002). Evolution of the Social Network of Scientific Collaboration. Physica A, Ireland, Departament of Chemistry, University College Dublin, Año 311, $\mathrm{N}^{\circ}$ 3-4, pp 590-614.

Beuren, Ilse Maria; Schlindwein, Antônio Carlos; Pasqual, Dino Luiz (2007) Abordagem da controladoria em trabalhos publicados no EnANPAD e no Congresso USP de controladoria e contabilidade de 2001 a 2006. Revista Contabilidade \& Finanças, Año 18, № 45, Brasil, Universidade de São Paulo, pp 22-37.

Bromwich, Michael (1990). The case for strategic management accounting: the role of accounting information for strategy in competitive markets. Accounting, Organizations \& Society, Año $15, \mathrm{~N}^{\circ}$ 1, United Kingdom, pp 27-46.

Brown, Lawrence D.; Gardner, John C. (1985a). Applying citation analysis to evaluate the research contributions of accounting faculty and doctoral programs. The Accounting Review, $\mathrm{N}^{\circ}$ 45, The Accounting Review, United 
States of America, American Accounting Association, pp 262-277.

Brown, Lawrence D.; Gardner, John C. (1985b). Using citation analysis to assess the impact of journals and articles on contemporary accounting research (CAR). Journal of Accounting Research, $N^{\circ} 46$, United States of America, University of Chicago, pp 84-109.

Brown, Lawrence D.; Gardner, John C; Vasarhelyi, Milklos A. (1987) An analysis of the research contributions of Accounting, Organizations \& Society, 19761984. Accounting, Organizations \& Society, № 12, United Kingdom, pp 193-204.

Cardoso, Ricardo Lopes; Pereira, Carlos Alberto; Guerreiro, Reinaldo (2007). Perfil das pesquisas em contabilidade de custos apresentadas no EnANPAD no período de 1998 a 2003. Revista de Administração Contemporânea, Año 11, № 3, Brasil, Associação Nacional das PósGraduação em Administração, pp 177-198.

Carvalho, Karine Lima; Saraiva Júnior, Abraão Freires; Frezatti, Fábio; Costa, Reinaldo Pacheco da (2010). A contribuição das teorias do ciclo de vida organizacional para a pesquisa em contabilidade gerencial. RAM - Revista de Administração da Mackenzie (Online), Año 11, № 4, Brasil, Universidade Presbiteriana Mackenzie, pp 98-130.

Casanueva Roche, Cristoba; Escobar Pérez, Bernabé; Larrinaga González, Carlos (2007). Red social de Contabilidad en España a partir de los tribunales de tesis. Revista Española de Financiación y Contabilidad, Año XXXVI, N ${ }^{\circ}$ 136, España, AECA, pp. 707-722.

Cooper, Robert (1987). Does your company need a new cost system? Journal of Cost Management, Año 1, $\mathrm{N}^{\circ} 1$, United States of America, pp 45-49.
Covaleski, Mark A.; Dirsmith, Mark W.; Samuel, Sajay (1996). Managerial accounting research: the contributions of organizational and sociological theories. Journal of Management Accounting Research, $N^{\circ} 8$, United States of America, American Accounting Association, pp 1-35.

Crane, Diane (1972). Invisible Colleges. Diffusion of Knowledge in Scientific Communities, Chicago University Press, United States of America, University of Chicago.

Da Cruz, Ana Paula Capuano; Espejo, Márcia Maria dos Santos Bortolocci.; Gassner, Flavia Pozzera.; Walter, Silvana Anita (2010) Uma Análise do Desenvolvimento do Campo de Pesquisa em Contabilidade Gerencial sob a Perspectiva Colaborativa Mapeada em Redes Sociais. Revista Contabilidade Vista \& Revista, Año 1, 21, No 2, Brasil, Universidade Federal de Minas Gerais, pp 95-120.

Dent, Jeremy F. (1990). Strategy, organization, and control: some possibilities for accounting research. Accounting, Organizations \& Society, Año 15, No 1/2, United Kingdom, pp 3-25.

Duarte, Fábio; Frey, Klaus. (2008) Redes Urbanas. In: Duarte, Fábio; Quandt, Carlos; Souza, Queila. O Tempo Das Redes. São Paulo (Brasil), Perspectiva.

Emirbayer, Mustafa; Goodwin, Jeff (1994) Network analysis, culture and the problem of agency. American Journal of Sociology, Año 99, $\mathrm{N}^{\circ}$ 6, United States of America, University of Chicago, pp 1411-1454.

Engwall, Lars (1998) Research note: Asterix in Disneyland. Management scholars from France on the world stage. Organizations Studies, Año 19, № 5 , United States of America, pp. 863881 
Autores y citas en contabilidad de gestión en revistas de lengua española

Lunkes, Rogério João; Ripoll Feliu, Vicente M. y da Rosa, Fabricia Silva

Escobar, Bernabé Pérez.; Lobo, Antonio Gallardo; Martínez, Cristina María Rocha Peña (2005). La Investigación Empírica en Contabilidad de Gestión en España. Análisis de las Publicaciones Española. Revista Espanola de Financiación y Contabilidad, Año XXXIV, No 124, España, AECA, pp. $183-210$

Freeman, Linton (2000). La centralidad en las redes sociales. Clarificación conceptual. Política y Sociedad, № 33 , España, Universidad Complutense de Madrid, pp 131-148.

Freeman, Linton (2006). The Development of Social Network Analysis. Vancouver: Empirical Press.

Galaskiewicz, Joseph; Wasserman, Stanley (1994). Advances in social network analysis: research in the social and behavioral sciences. London: Sage.

Groot, Tom; Garcia-Valderrama, Teresa (2006). Research quality and efficiency - An analysis of assessments and management issues in Dutch economics and business research programs. Research Policy. Año 35, $\mathrm{N}^{\circ}$ 9, United States of America, $\mathrm{pp}$ 1362-1376

Hesford, James W.; Potter, Gordon (2010). Accounting Research in the Cornell Quarterly: A Review with Suggestions for Future Research. Cornell Hospitality Quarterly. Año, 51, № 4, United States of America, Cornell University, pp 502-512.

Hesford, James W., Lee, Sung-Han; Van Der Stede, Wim A.; Young, S. Mark (2007). Management accounting: a bibliographic study. In: Chapman, Christopher; Hopwood, Anthony G.; Shields, Michael (Org.). Handbook of management accounting research. Amsterdam (Netherlands): Elsevier.

Hiromoto, Toshiro (1988). Another hidden edge: Japanese management ac- counting. Harvard Business Review, Año, 66, № 4, United States of America, Harvard Business School, pp 22-25.

Hopwood, Anthony G. (1978). Towards an organizational perspective for the study of accounting and information systems. Accounting, Organizations \& Society, Año, 3, № 1, United Kingdom, pp 3-13.

Johnson, Thomas, H.; Kaplan, Robert S. (1987). Relevance lost: the rise and fall of management accounting. Boston, MA: Harvard Business School Press.

Kaplan, Robert S.; Norton, David P. (2001). Organização orientada para a estratégia. Rio de Janeiro: Campus, Brasil.

Kaplan, Robert S. (1983). Measuring manufacturing performance: a new challenge for management accounting research. The Accounting Review, Año 58, № 4, United States of America, American Accounting Association, pp 686-705.

Kaplan, Robert S.; Norton, David P. (1992). The balanced scorecard: measures that drive performance. Harvard Business Review, Año 70, № 1, United States of America, Harvard Business School, pp 71-79.

Katz, Sylan; Martin, Ben (1997). What is research collaboration? Research Policy, $N^{\circ} 26$, United States of America, pp 1-18.

Kirkpatrick, Shelley.; Locke, Edwin. (1992). The Development of Measures of Faculty Scholarship. Group \& Organization Management. Año 17, $\mathrm{N}^{\circ}$ 1, United States of America, University of West Florida, pp 5-23.

Kuhn, Thomas (1962). La Estructura de las Revoluciones Científicas. FCE, México. 
Larrinaga, Carlos (2005). Producción Científica en Contabilidad: El Caso de España (1992-2002). Revista Española De Financiación Y Contabilidad, Año XIX, No 125, España, AECA, pp. 363-393

Lunkes, Rogério João; Ripoll Feliu, Vicente Mateo; Rosa, Fabricia Silva (2011). Redes Sociais e Internacionalização Da Contabilidade Gerencial: Um Estudo em Publicações de Lingua Espanhola. Congresso de Contabilidade e Auditoria, Año 1, No 1, Porto (Portugal), pp.1-15.

Macintosh, Norman; Scapens, Robert (1990). Structuration theory in management accounting. Accounting, Organizations \& Society, Año 15, № 5, United Kingdom, pp 455-477.

Malmi, Teemu; Granlund, Markus (2009). In Search of Management Accounting Theory. The European Accounting Review. Año 18, № 3, Belgium, European Accounting Association, pp 597-620.

Maloni, Michael; Carter, Craig; Carr, Amelia (2009) Assessing logistics maturation through author concentration. International. Journal of Physical Distribution \& Logistics Management. Año 39, № 3- of Alabama, pp 250-268. 4, United States of America, The University

Molina, José Luis; Muñoz, Juan Manoel; Domenech, Miquel (2002) Redes de publicaciones científicas: Un análisis de la estructura de coautorías. Redes. Revista Hispana para el Análisis de Redes Sociales, Enero, Año 001, N 3 Universidad Autónoma de Barcelona, España, pp 1-15.

Moody, James (2004) The Structure of a Social Science Collaboration Network: Disciplinary Cohesion from 1963-1999. American Sociological Review, Año 69, No 2, United States of America,
American Sociological Association, pp 213-239.

Nascimento, Artur Roberto; Junqueira, Emanuel; Martins, Gilberto de Andrade (2010) Pesquisa acadêmica em contabilidade gerencial no Brasil: análise e reflexões sobre teorias, metodologias e paradigmas. Revista de Administração Contemporânea. Año 14, $\mathbf{N}^{\circ}$ 6, Brasil, Associação Nacional de Pós-Graduação em Administração, pp 1113-1133.

Newman, Mark.E.J. (2001) Scientific collaboration networks. I. Network construction and fundamental results. Physical Review. $\mathbf{N}^{\circ}$ 64, United States of America, American Physical Society, pp16-31.

Owen-Smith, Jason; Powell, Walter (2008) Networks and Institutions. In: Greenwood, R.; C. Oliver; K. SahlinAndersson $Y$ R. Suddaby, The sage handbook of organizational institutionalism. London: Sage Publications, pp 594-621.

Rossoni, Luciano; Machado-Da-Silva, Clóvis (2007) A construção social do conhecimento em campos científicos: análise institucional e a configuração de mundos pequenos. EnAMPAD. Anais (CD-ROM). Rio de Janeiro (Brasil): ANPAD.

Salas, Oriol Amat; Blake, John; Gowthorpe, Catherine; Sobrevia, Ester Oliveiras (1998). Análisis de Autores, Citas y Revistas de Contabilidad en España. Revista Española De Financiación y Contabilidad Año XXVII, Nº 96, España, AECA, pp $821-850$

Scapens, Robert (2004). Innovations in management accounting practices and research in the United Kingdom. Keynote address at the 4th New Directions in Management Accounting: Innovations in Practice and Research conference, EIASM, Año 9, Brussels (Belgium), pp 9-11. 
Autores y citas en contabilidad de gestión en revistas de lengua española

Lunkes, Rogério João; Ripoll Feliu, Vicente M. y da Rosa, Fabricia Silva

Scott, Richard (2008). Institutions and organizations: ideas and interests. 3 . ed.: New York (EUA): Sage Publications.

Shank, John.; Govindarajan, Vijay (1993). Strategic cost management: the new tool for competitive advantage. New York (EUA): The Free Press.

Shields, Michael (1997). Research in management accounting by North Americans in the 1990s. Journal of Management Accounting Research, № 9 , United States of America, American Accounting Association, pp 3-61.

Simons, Robert (1995). Levers of Control How Managers Use Innovative Control Systems to Drive Strategic Renewal. Boston (EUA): Harvard Business School.

Tahai, Alireza; Meyer, Michael (1999). A revealed preference study of management journals' direct influences. Strategic Management Journal. Año 20, $\mathrm{N}^{\circ} 3$, pp 279-296.

Valacich, Joseph.; Fuller, Mark.; Schneider, Christoph; Dennis, Alan (2006). Is- sues and opinions - Publication opportunities in premier business outlets: How level is the playing field? Information System Research. Año 17, $\mathrm{N}^{\circ} 2$, United States of America, Institute for Operations Research and the Management Science, pp 107-125.

Wasserman, Stanley; Faust, Katherine (1994). Social network analysis: methods and applications. Cambridge, UK: Cambridge University Press.

White, Gregory (1996). A survey and taxonomy of strategy-related performance measures for manufacturing. International Journal of Physical Distribution \& Logistics Management. Año $16, N^{\circ} 3$, United States of America, The University of Alabama, pp 42-68.

Young, Mark; Selto, Frank (1991). New manufacturing practices and cost management: a review of the literature and directions for future research. Journal of Accounting Literature, $\mathrm{N}^{\circ} 10$, United States of America, Warrington College of Business Administration, University of Florida, pp 265-298. 